\title{
Role of Serratiopeptidase in Enzyme Therapy
}

\author{
Sri Neeraja .P \\ Intern, Saveetha Dental College, Chennai, India
}

\begin{abstract}
Enzymes are essential for each and every reaction in a living organism. They are necessary for adequate functioning of our whole metabolism. Enzymes often provide a means of monitoring a patient's response to therapy.Therapeutic use of natural enzyme is known as Enzyme therapy. They have even become one of the most innovative and expanding drug groups, and systemic therapy with proteolytic enzymes has become an important method of treatment in natural medicine. Serratiopeptidase is an enzyme derived from bacteria Enterobacteria serratia isolated from intestine of silkworms.Serratiopeptidas is used as an anti-inflammatory medicine.The activities of Serratiopeptidase isseen in this review. Serratiopeptidase has revealed new and more interesing applications in the field of pain management and transferance of antibiotics.
\end{abstract}

Keywords: Serratiopeptidase, anti-inflammatory, enzyme, pain

\section{Introduction}

Enzymes are biocatalyst which produce, with a small effort, a big effect. They are constantly produced within ourbody. The application of enzyme technologies to pharmaceutical research, development and manufacturing is a growing field.Enzyme therapy came into use around 1960s.[1]

Inflammation is the first step of healing process and it is the initial response towards injury, thus serves as a mechanism to protect our body. But inflammation can cause pain and damage to the body.[2]

Serratiopeptidase is a protiolytic enzyme excreted by nonpathogenic Enterobacteria serratia isolated from intestine of silkworms Bombyx mori L. It has a distinctive ability to digest the dead tissue of the Cocoon of the silkworm.[4] A grayish white to pale brown colour powder with characteristic odour.

It is widely recognised throughout Asia, Europe,US.In 1957 Japanese began using Serratiopeptidase for inflammation.[3]In human body it breaks down protein deposits such as fibrin. This is used as a natural alternative to steroids and NSAIDs without serious side effects. The enzyme causes proteolysis of all non-vital tissues including blood clots, cysts, tissue plaques and cellular debris and reduces the inflammatory response.

Serratiopeptidase binds to $\alpha_{2}-$ macroglobulin in the blood in $1: 1$ ratio. It is slowly transferred to the exudates at the site of infection and inflammation. Mechanism of action is by hydrolysis of histamin, bradykinin and serotonin. By hydrolyzing bradykinin, histamine and serotonin it indirectly reduces dilatation of blood capillaries and controls permeability. Serratiopeptidase blocks plasmin inhibitors thus helping the fibrinolytic activity of plasmin.Degradation of extra-fibrin to small fragment prevent clogging of microcapillaries, helps clearance of exudates, reduces swelling and improves microcirculation. \& expectoration of sputum etc.

Serratiopeptidase has been effective in trasferring various antibiotics like Penicillin G, Ampicillin, Gentamicin or
Cefotaxim to the site of infection.[13] They have various activities like caseinolytic activities, fibrinolytic activities.

It was found that this enzyme is used for pain, inflammation due to arthritis, trauma, surgery, sinusitis,bronchitis,carpal tunnel and painful swelling of the breasts. Serratiopeptidase is stored at cool temperature and kept away from direct sunlight. They are available as enteric coated Serratiopeptidase granules.[16] On oral administration, serratiopeptidase is absorbed in GI tract and distributed throughout the body tissues unchanged via systemic circulation.[15]

Serratiopeptidase has few disadvantages as it is susceptible to degradation and vulnerable to acidic $\mathrm{pH}$. The adverse effects may include nausea, vomitting, diarrhoea, epistaxis, haemoptysis and sometimes hypersensitivity.This increased risk of bleeding may also occur when this enzyme is taken with other natural remedies such as garlic, fish oil and turmeric.[14]

\section{Serratiopeptidase for inflammation:}

It acts in three ways in reducing the inflammation: it breaks down insoluble protein byproducts of blood coagulation, thins the fluids formed from inflammation and injury and facilitates the rate of tissue repair process. They reduce pain by blocking the pain inducing amines.It also dissolves the dead tissue surrounding the injured area and promotes healing.[5] Serratiopeptidase also works by modifying cellsurface adhesion molecules.Unlike NSAIDs pain medications, Serratiopeptidase does not cause dangerous internal bleeding and it is not addictive. They are used for the purpose of suppression and elemination of post operative bleeding, haematoma.[6]

\section{Serratiopeptidase for Sinusitis and Bronchitis:}

Inflammation is seen in the lining of the air way passage that prevent drainage of mucus during these two conditions. Obstruction is seen as the mucus in that area becomes thick. Serratiopeptidase has strong anti-inflammatory and antitumefacient effect. It acts by elemination of inflammatory oedema and swelling, acceleration of liquefaction of pus and sputum and enhancement of the action of antibiotics.[11]Serratiopeptidase alters the elasticity of the mucus by reducing the thickness and viscosity by 


\section{International Journal of Science and Research (IJSR) \\ ISSN (Online): 2319-7064 \\ Index Copernicus Value (2015): 78.96 | Impact Factor (2015): 6.391}

elimination of bronchopulmonary secretions and does not deplete the mucus.[8]

\section{Serratiopeptidase for carpal tunnel syndrome:}

Carpal tunnel syndrome is an inflammatory disorder of hand and wrist causing intense long lasting pain, inflammation and disability.Serratiopeptidase has an effect on this syndrome without causing side effects. It was proven to be effective at reducing swelling and pain intensity in individuals with carpel tunnel and sprained ankles.[6][9]

\section{Serratiopeptidase for infection}

Bacteria can produce biofilm which causes resistance to antibiotic. Serratiopeptidase enhances the effectiveness of antibiotics (ampicillin,cephalexin, cefotim) against biofilm and inhibits its formation. It acts as a adjuvant in infections such as alveolar abscess, periodontitis, septic abortion, salpingitis, pulmonary tuberculosis, etc. [11] It acts on the plaque accumulation in the arteries. It removes the deposits of fatty substances, cholesterol, cellular waste products, calcium and fibrin in side the artery. The anti-inflammatory activity helps in breast engorgements and pregnancy related thromboplbitis. Serratiopeptidase restores microcirculation and increases antibiotic penetration in male genital organs. [7]

\section{Conclusion}

The use of enzymes in medical therapy has been limited. This Serratiopeptidase has a remarkable record of safety from decades of use by million of users all around the world.The recommended dosage for Serratiopeptidase is 10 $\mathrm{mg}$ to $30 \mathrm{mg}$ a day. Serratiopeptidase is well known for its ability to reduce pain by blocking the release of paininducing molecules from inflamed tissues. [10] As enzymes bind and act on their targets with greater affinity and specificity, convert multiple target molecules to desired products, it has resulted in the development of enzyme specific drugs for a wide range of disorders.

\section{References}

[1] John Beard, Herxheimer, Inderst, Kleine, Kunz,Jaeger, Seifert, Hector E. Solorzano del Rio, Wolf, Wrba; Systemic Enzyme Therapy. http://www.arthritistrust.org/Articles/Systemic\%20Enzy me\%20Therapy/ .

[2] Michel Vellard;The enzyme as drug: application of enzymes as pharmaceuticals;Current Opinion in Biotechnology 2003, 14:444-450

[3] Mairi R. Ross, Health Writer ,Mark Cho; Serratiopeptidase-The Miracle Enzyme.http://leadspharma.in/pdf/SerratiopeptidaseThe-Miracle-Enzyme.pdf.

[4] Miyata K; Intestinal absorption of Serratia Peptidase. J Appl Biochem. 1980;2:111-16.

[5] Mazzone A, Catalani M, Costanzo M, Drusian A, Mandoli A, Russo S, Guarini E, Vesperini G. Evaluation of Serratia peptidase in acute or chronic inflammation of otorhinolaryngology pathology: a multicentre, double-blind, randomized trial versus placebo. J Int Med Res. 1990;18(5):379-88.
[6] Esch PM, Gemgross H, Fabian A..Reduction of postoperative swelling.Objective measurement of swelling of the upper ankle joint in treatment with serrapeptase - a prospective study (German) 2FortscherMed. 1989; 107(4):67-8.

[7] Kee WH, Tan SL, Lee V, Salmon YM. The treatment of breast engorgement with Serrapeptase (Danzen); a randomized double-blind controlled trial. Singapore Med J. 1989;30(1):48-54.

[8] Majima Y, Inagaki M, Hirata K, Takeuchi K, Morishita A, Sakakura Y. The effect of an orally administered proteolytic enzyme on the elasticity and viscosity of nasal mucus. Arch otorhinolaryngol. 1988;244(6):3559.

[9] Panagariya A, Sharma AK. A preliminary trial of serratiopeptidase in patients with carpal tunnel syndrome. J Assoc Physicians India; 1999; 47 (12); 1170-1172.

[10]Dr. David Jockers, SerratioPeptidase is a potent antiinflammatory enzyme at http://www.naturalnews.com.

[11] Mecikoglu M, Saygi B, Yildirim Y, Karadag-Saygi E, Ramadan SS, Esemenli T. The effect of proteolytic enzyme serratiopeptidase in the treatment of experimental implant-related infection. J Bone Joint Surg Am. 2006 Jun;88(6):1208-14.

[12] Nakamura S, Hashimoto Y, Mikami M, Yamanaka E, Soma T, Hino M, Azuma A, Kudoh S. Effect of the proteolytic enzyme serrapeptase in patients with chronic airway disease. Respirology. 2003 Sep;8(3):316-20.

[13] Okumura H, Watanabe R, Kotoura Y, Nakane Y, Tangiku O; Effects of a proteolytic enzyme preparation used concomitantly with an antibiotic in osteoarticular infection; Jpn J Antibiot 1977 Mar;30(3):223-7.

[14] Serratiopeptidase/Serratia Peptidase Side Effects and Health Benefits at. http://www.pharmaceutical-drugmanufacturers.com

[15] Malshe PC. Orally administered serratiopeptidase: can it work? J Assoc Physicians India 1998; 46(5):492.

[16] Kalpesh K. Joshi And Rajan P. Nerurkar; AntiInflammatory Effect Of The Serratiopeptidase Rationale Or Fashionable: A Study In Rat Paw Oedema Model Induced By The Carrageenan; Indian J PhysiolPharmacol 2012; 56(4) : 367-374. 